\title{
Optimizing GC-MS Analysis of 3-MCPD and Glycidyl Esters
}

Jana Rousova, Joe Konschnik, Hansjoerg Majer, Alexandria Pavkovich and Chris English Restek Corporation, 110 Benner Circle; Bellefonte, PA, USA

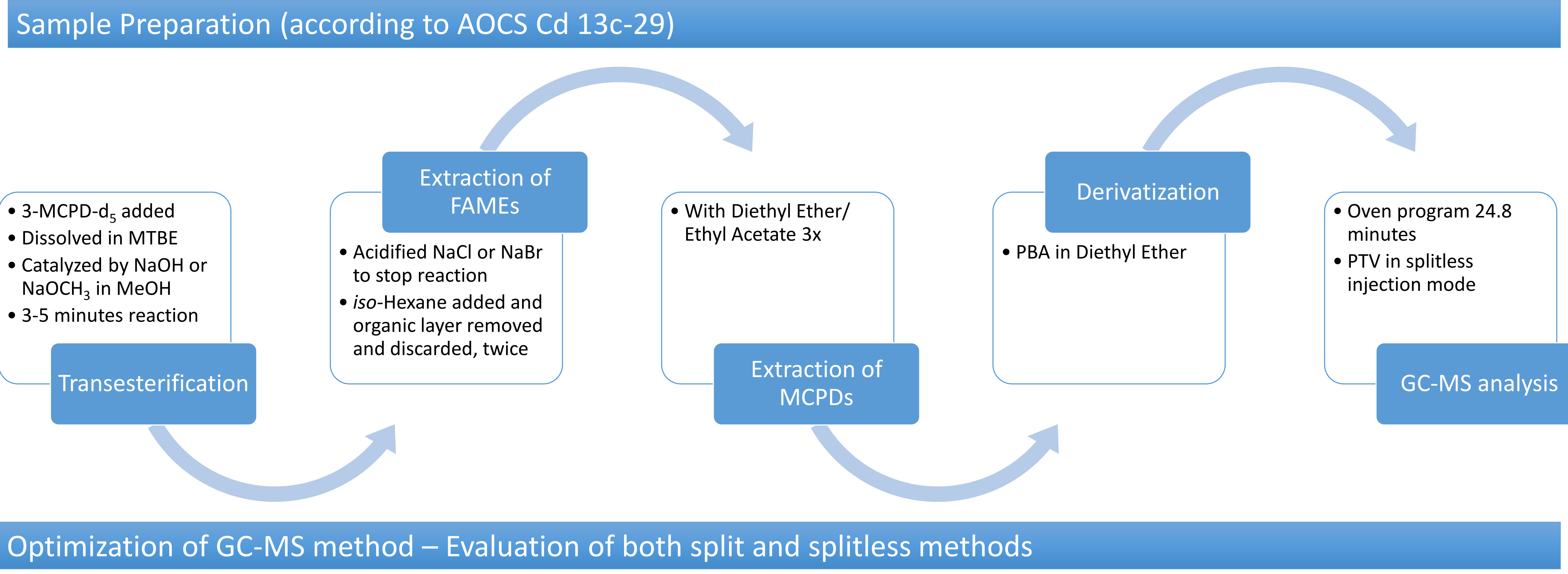

3-MCPD and glycidyl esters in edible oils are contaminants that are formed through refining processes. Several of these substances have been classified as possible human carcinogens. Methods, which are similar to one another, have been developed by ISO, AOCS, and DGF for analyzing these contaminants. While these methods cover extraction and derivatization techniques in detail, very little attention is paid to the GC-MS methods. With emerging automated systems, it is important to simplify and speed up the instrument method by optimizing the parameters, to include evaluating split injection.

\section{Optimization of GC-MS method}

\begin{tabular}{|c|c|c|r|r|c|}
\hline Initial temp. $\left({ }^{\circ} \mathrm{C}\right)$ & & & & \\
\hline Splitless & $\mathrm{RT1}(\mathrm{min})$ & $\mathrm{RT2}(\mathrm{min})$ & Width1 & Width2 & Resolution \\
\hline 95 & 8.03 & 8.07 & 0.030 & 0.035 & 0.762 \\
\hline 100 & 7.62 & 7.66 & 0.022 & 0.034 & 0.864 \\
\hline 105 & 7.22 & 7.26 & 0.023 & 0.021 & 1.126 \\
\hline 110 & 6.82 & 6.86 & 0.023 & 0.022 & 1.075 \\
\hline 115 & 6.42 & 6.46 & 0.025 & 0.025 & 0.944 \\
\hline 120 & 6.04 & 6.07 & 0.027 & 0.027 & 0.830 \\
\hline Split & & & & & \\
\hline 95 & 8.03 & 8.07 & 0.020 & 0.028 & 1.033 \\
\hline 100 & 7.62 & 7.66 & 0.020 & 0.019 & 1.241 \\
\hline 105 & 7.22 & 7.26 & 0.021 & 0.019 & 1.209 \\
\hline 110 & 6.82 & 6.86 & 0.019 & 0.019 & 1.273 \\
\hline 115 & 6.43 & 6.47 & 0.019 & 0.019 & 1.242 \\
\hline 120 & 6.04 & 6.0 & 0.019 & 0.018 & 1.244 \\
\hline
\end{tabular}

Comparison of calibration curves for both split and splitless injections

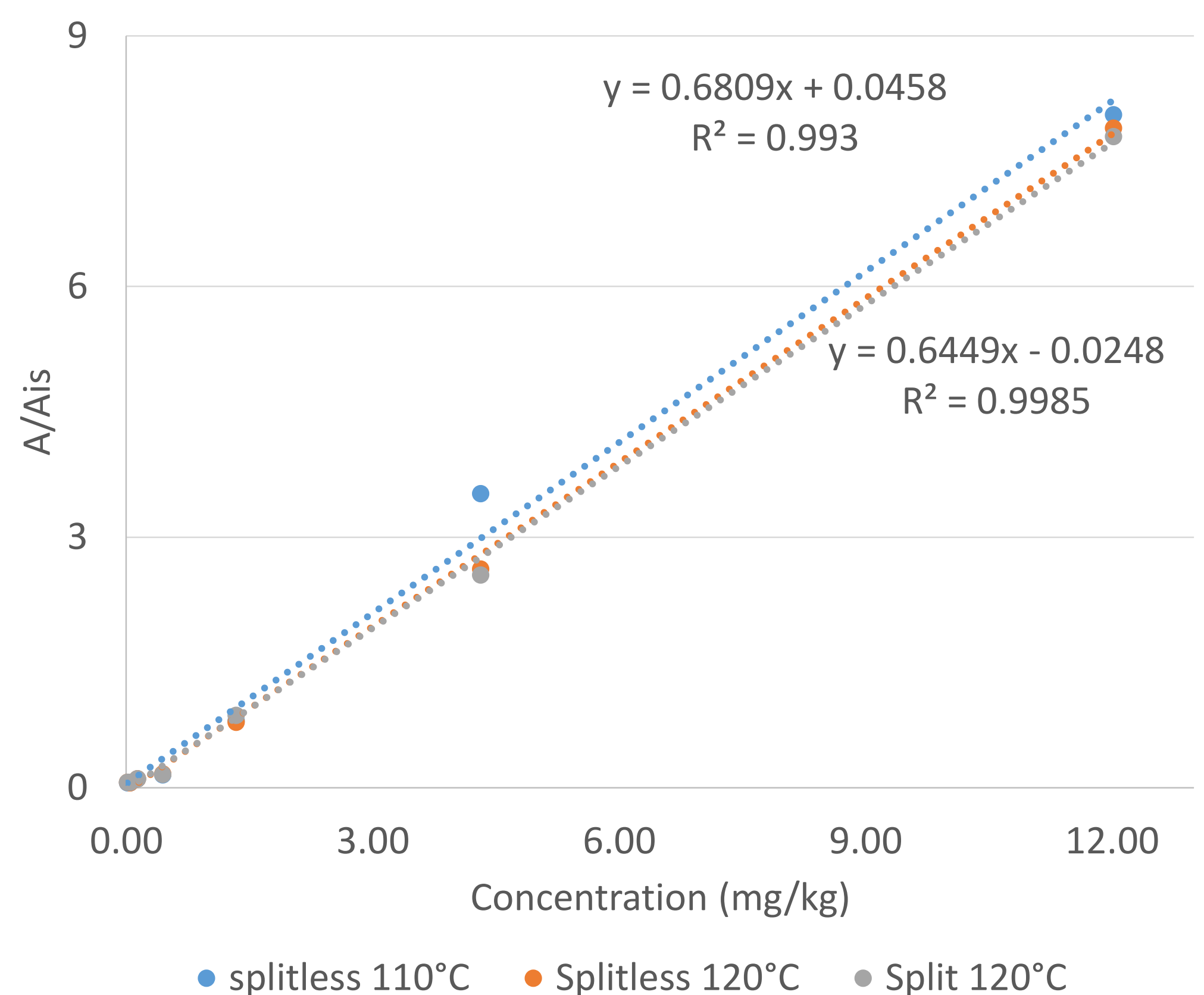

Comparison of calibration curves for split injection at different temperatures/inlets

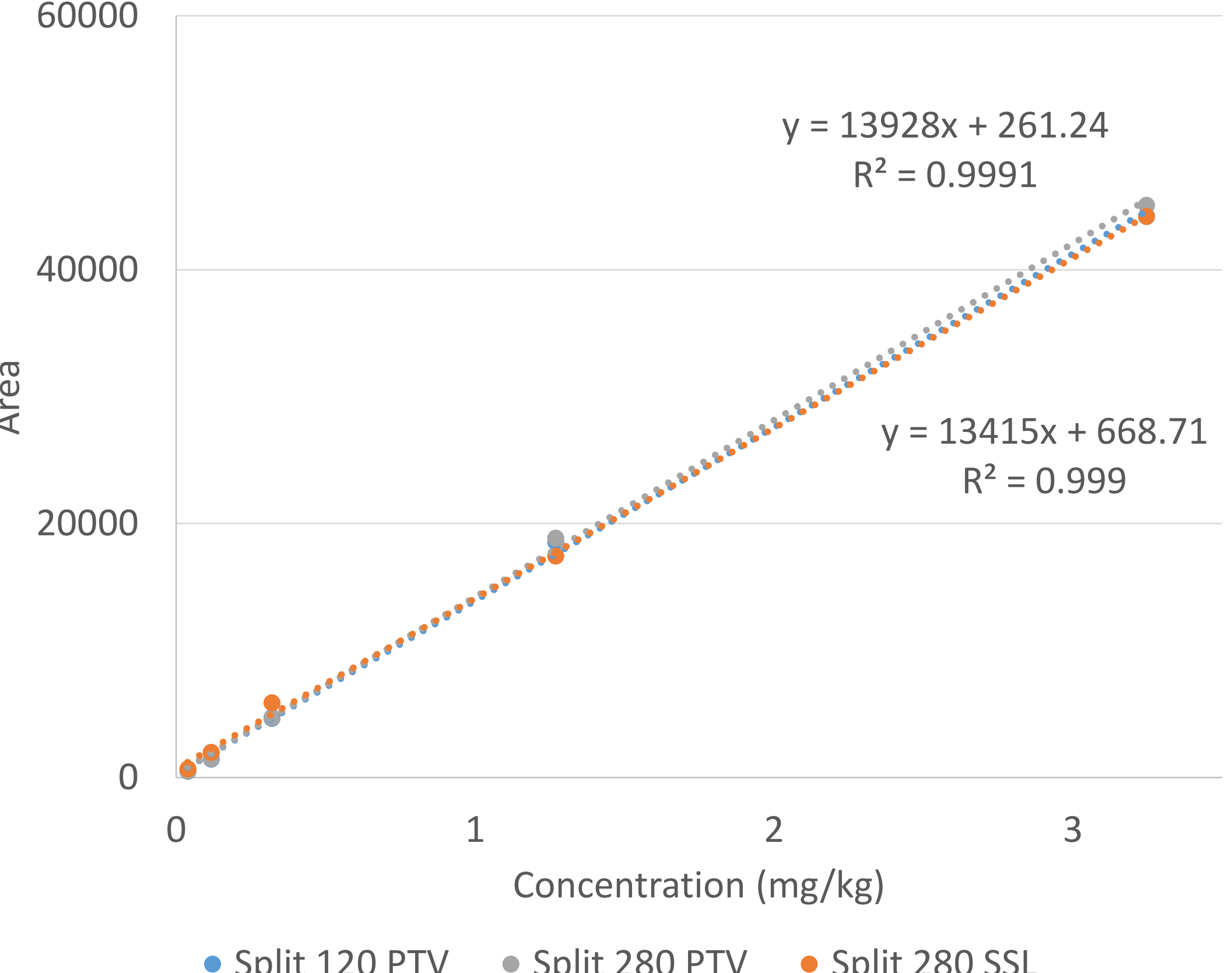

ProEZGC Model on Rxi-17Sil MS 30x0.25x0.25

\section{Pro EZGC}

\section{Chromatogram Modele}

Pro EZGC is a free GC modeler that allows for fast and simple optimization our analysis Compounds currently present in the system: 3-MCPD and 3-MCPD-d5 derivatized with PBA

2-MCPD and 2-MCPD-d5 derivatized with PBA

lycidyl and glycidyl-d5 as 3-MBPD and 3-MBPD-d5 (resp.) derivatized with PBA

Phenyl boronic acid (PBA)

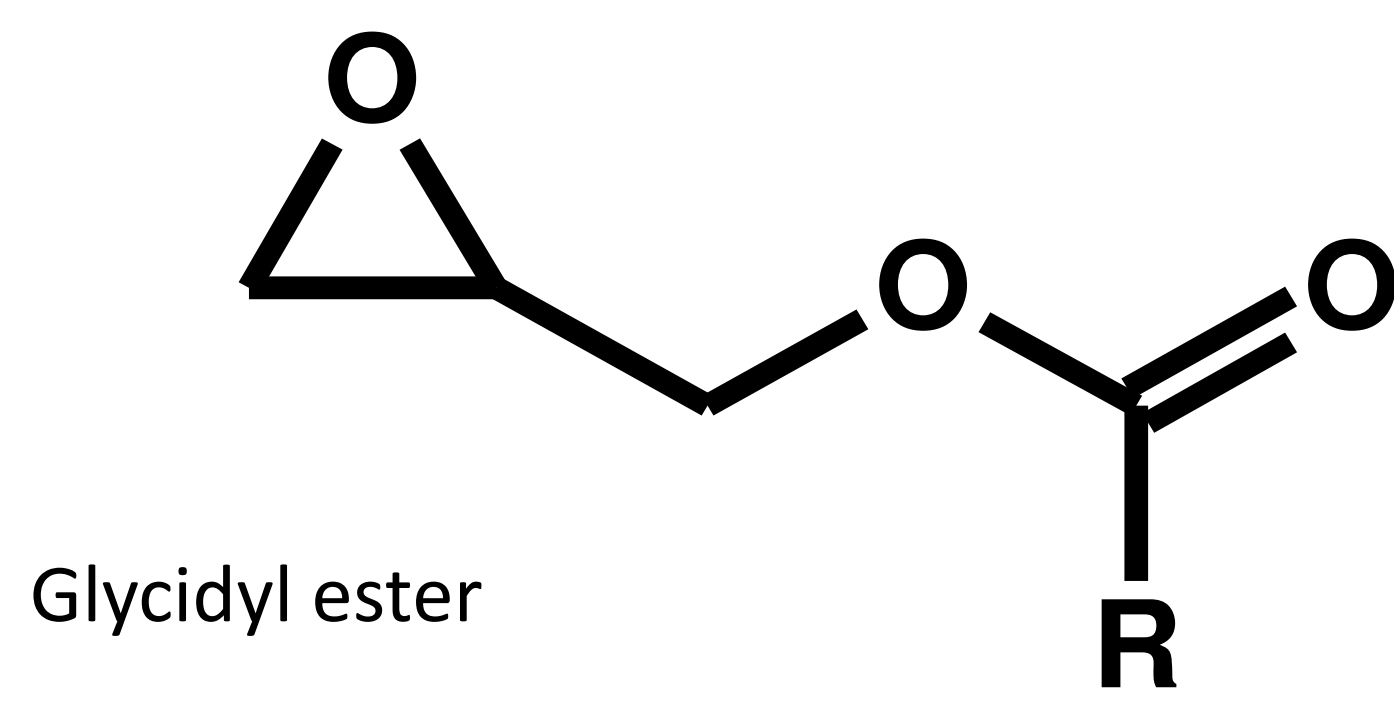<smiles>[R]C(=O)OCC(CCl)OC([R])=O</smiles>

\section{Changing the GC-MS Method}

Original method: New, optimized method: Temp program: $85^{\circ} \mathrm{C}(0.5)$, Temp program: $120^{\circ} \mathrm{C}(0.5)$ $6^{\circ} \mathrm{C} / \mathrm{min}$ to $150^{\circ} \mathrm{C}$ $12^{\circ} \mathrm{C} / \mathrm{min}$ to $180^{\circ} \mathrm{C}$ $25^{\circ} \mathrm{C} / \mathrm{min}$ to $280^{\circ} \mathrm{C}(7)$ total time: $24.8 \mathrm{~min}$ Splitless time $0.5-1 \mathrm{~min}$

\section{Conclusions}

- An optimized GC-MS method led to improved peak shapes without detrimental effect on resolution. Manually optimized temperature program saved 8 minutes per analysis. EZGC model can save up to $\mathbf{2 0}$ minutes.

- Switching to split injection had no negative effect on limits of detection.

- Using regular split/splitless injector had no effect on the performance. However, using guard column is recommended. to $330^{\circ} \mathrm{C}(5)$; total time: 16.5 $\min$

Split 10:1

\section{MCPD and GE analysis}

Rxi-17Sil MS 30mx0.25x0.25

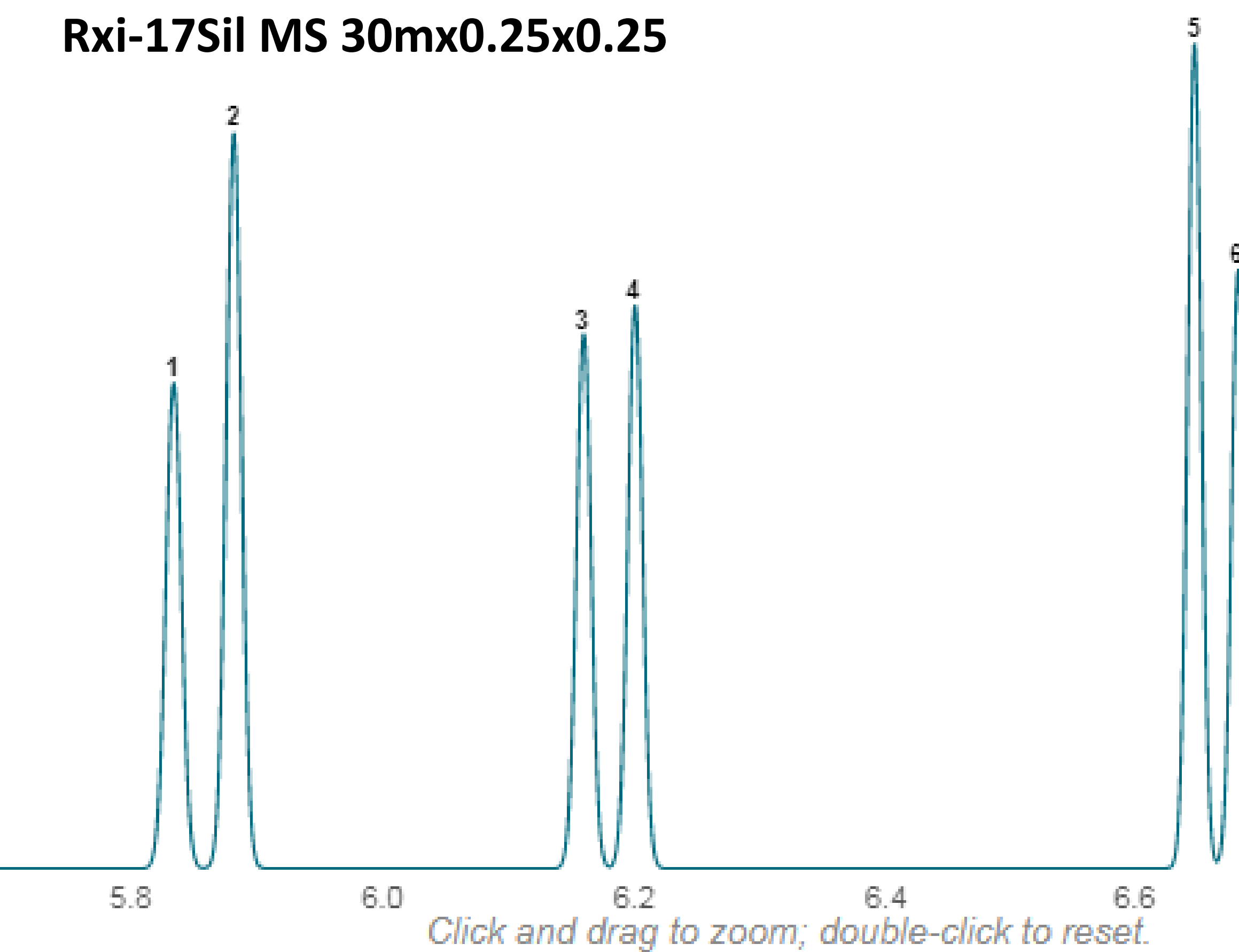

Column: Rxis-17Sil MS, $30.00 \mathrm{~m}, 0.25 \mathrm{~mm}$ ID, $0.25 \mathrm{~mm}$ (cat. 14123 )

\section{Carrier Gas: Helium, Constant Flow $91.40 \mathrm{~mL} / \mathrm{min}$}

Oven Temp: $120^{\circ} \mathrm{C}$ (hold $0.5 \mathrm{~min}$ ) to $180^{\circ} \mathrm{C} @ 12^{\circ} \mathrm{C} / \mathrm{min}$ to $330^{\circ} \mathrm{C} @ 25^{\circ} \mathrm{C} / \mathrm{min}$

\begin{tabular}{|c|c|c|c|c|c|}
\hline Peaks & & $\left.\begin{array}{c}\stackrel{t}{R}_{R} \\
(\mathrm{~min}\end{array}\right)$ & $\mathbf{R}_{\mathbf{s}}$ & $\begin{array}{l}\text { Peak } \\
\text { Width } \\
\text { (min) }\end{array}$ & $\begin{array}{l}\mathrm{T}_{\text {peek }} \\
\left({ }^{\circ} \mathrm{C}\right)\end{array}$ \\
\hline 1. 3-MCPD-d5 & Q & 5.83 & 1.7 & 0.028 & \\
\hline 2. 3-MCPD & a & 5.88 & 1.7 & 0.027 & 189.5 \\
\hline 3. 2-MCPD-C & a & 6.16 & 1.5 & 0.026 & 196 \\
\hline 4. 2-MCPD & a & 6.20 & 1.5 & 0.026 & 197 \\
\hline 5. Glycidyl-d5 & Q & 6.65 & 1.4 & 0.025 & 208 \\
\hline 6. Glyci & a & 6.68 & 1.4 & 0.024 & 200 \\
\hline 7. Phenylboro & a & 10.61 & 161 & 0.021 & 307 \\
\hline
\end{tabular}
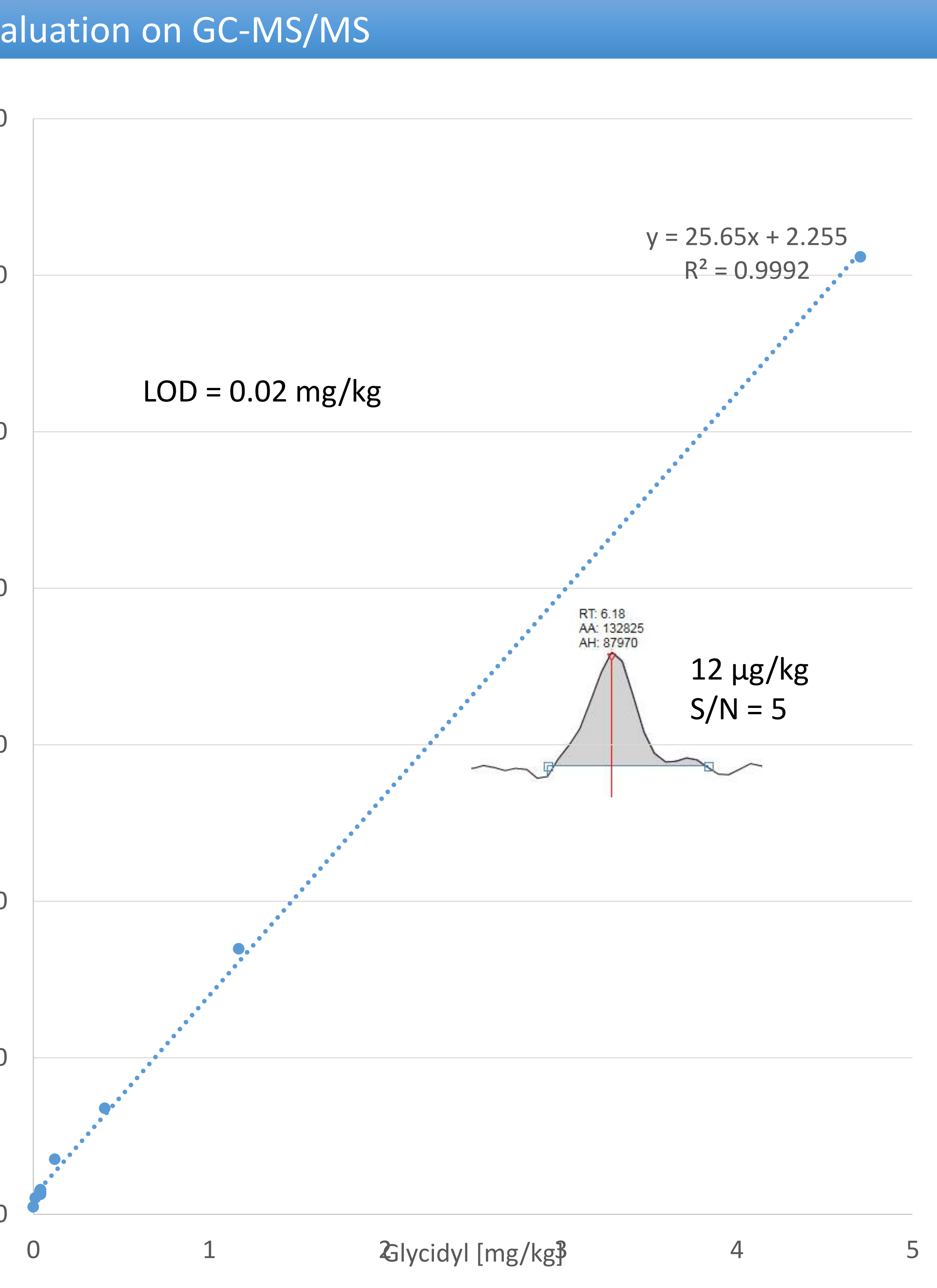

\section{Fast Analysis on Rxi-!7Sil MS 20x0.18x0.18 - EZGC and Evaluation}

Fast MCPD and GE analysis

Rxi-17Sil MS 20x0.18x0.18

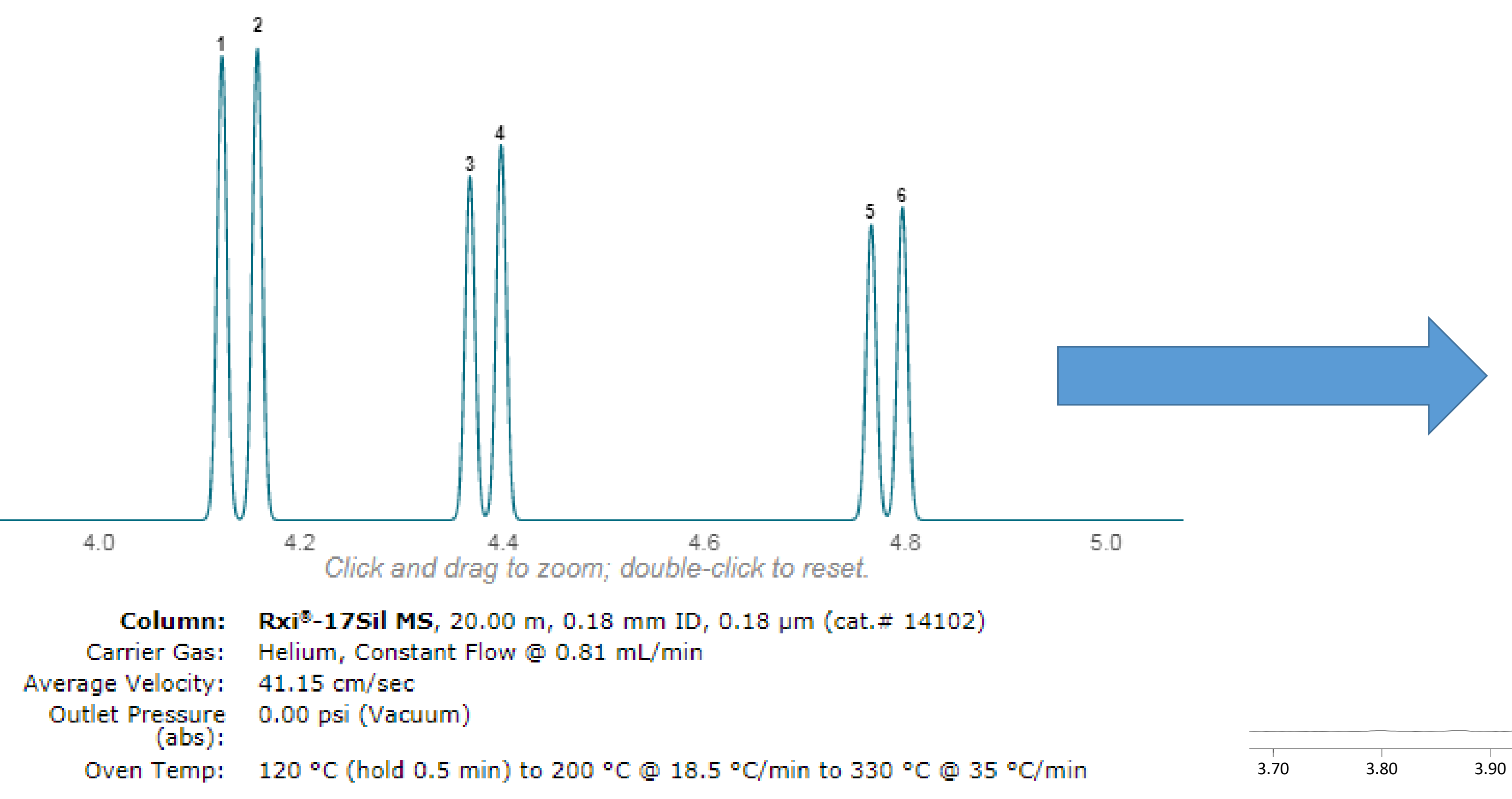

